\title{
Author Index to Volume 11
}

(BR) Book review; (C) Corrigendum; (CP) Course profile; (CSR) Case report; (E) Editorial; (ER) Erratum; (GP) Genetic policy; (L) Letter; (MR) Meeting Report; (PG) Practical genetics; (R) Review; (SR) Short report; (VP) Veiwpoint

Abad C 879 (SR)

Abbasi AA 812 (SR)

Abdalla SA 279

Abe S 744, 916

Abolmasov NN 444

Achmann R 913 (L)

Adamovic S 828

Aghaie A 816 (SR)

Agtmael TV 779

Ahmad W 77 (SR), 623 (SR), 812 (SR)

Ainsworth P 951

Akey J 39 (SR)

Ala-Kokko L 185, 265

Albrecht B 858

Alchorne MMA 64 (SR)

Alfano G 155

Almeida R 380

Almer S 179

Alnaes GG 416 (SR)

Amin ud Din M 77 (SR)

Amos $\mathrm{Cl} 425$

An J 714 (SR)

Anderlid B-M 89 (SR)

Andley UP 784

Andreassi MG 671

Andréo B 337

Andrés AM 385

Andreussi L 245

Andriulli A 687

Anetseder MJ 342 (SR)

Angelini C 710 (SR)

Ansar M 77, 623, 812 (SR)

Antoccia A 297

Antson D-O 357

Arends J 611

Armengod ME 959

Armour JAL 911 (L)

Aro AR 565

Arshad M 77 (SR)

Arte S 866

Ascaso JF 959

Ascher H 828

Asmus F 138

Asplund K 81 (SR)

Assael BM 93 (SR), 543 (SR)

Ataie A 816 (SR)

Aulehla-Scholz C 913 (L)

Aunger R 253

Avella AMB 409
Avidan N 497

Avril M-F 288

Ayadi H 185

Ayme S 899 (E), 900, 903, 906, 909 (E)

Babron M-C 828

Baiget M 489 (SR)

Bailey-Wilson JE 835

Bairead E 237

Bais AG 409

Balanovskaya EV 444

Balcı S 851

Balg S 858

Banfi S 155

Bannister W 437

Barać L 535

Barbet F 966 (SR)

Barker KT 665

Barrantes R 39 (SR)

Barthelme A 497

Barwell J 749

Basso C 69 (SR)

Bastert G 464

Battelino T 97 (SR)

Bauce B 69 (SR)

Baumann C 452

Baumer A 913 (L)

Bayoumi R 39 (SR)

Beaty TH 835

Bebyakova NA 444

Bechner L 585

Becker T 637

Beckmann JS 497

Beekman M 845

Beffagna G 69 (SR)

Belaiche J and the GETAID 179

Belpinati F 93 (SR), 553 (L)

Ben-Asher E 497

Bencivenga P 503

Bene J 375

Benet J 325, 879 (SR)

Benítez J 489 (SR)

Bennetts BH 509, 945

Bennum R 835

Bentley GR 253

Benzacken B 452

Bergen AAB 215
Bertoni C 210

Bertranpetit J 385

Besse-Schmittler C 590

Bétard C 770

Bets LV 444

Beumont PJV 945

Biagini A 671

Bint S 749

Bisignano G 433

Blennow E 89 (SR)

Blom HJ 23 (SR)

Blom J 633

Blomstrand C 603

Bo RD 896 (SR)

Boers GHJ 23 (SR)

Boerwinkle E 271 (SR), 425

Böhringer S 573

Boiso I 325

Bombieri C 553 (L)

Bonamini D 93 (SR), 543 (SR)

Bonne-Tamir B 760

Boogaerts A 30 (SR)

Boomsma DI 845

Booth DR 509

Bordoni A 896 (SR)

Borot N 497

Børresen-Dale A-L 416 (SR)

Bosch M 754

Bosse K 643

Botto N 671

Bougeard G 700 (SR)

Braithwaite AW 840

Brancadoro V 342 (SR)

Brancati F 972 (SR)

Brauer S 304

Bravo LE 380

Breedveld G 409

Brekelmans CTM 633

Bresolin N 896 (SR)

Bressac-de Paillerets B 288

Briault S 352

Briuglia S 433

Broeckhoven CV 779

Bruni AC 597

Brunner HG 57 (SR), 884 (SR)

Brunoni D 64 (SR)

Buiting K 913 (L)

Bungardt N 464

Bürger J 913 (L)

Burwinkel B 516
Caccioppoli C 155

Calafell F 385

Calda P 835

Caldés T 489 (SR)

Calevo MG 245

Calvas P 163

Cama A 245

Camaschella C 585

Cambien F 659

Cambon-Thomsen A 475

Camp GV 744

Campillo M 325

Capra V 245

Cardon L 638 (L)

Cardoso C 527

Carella M 585

Carlsson B 189

Carmena R 959

Carracedo A 489 (SR)

Carsana A 342 (SR)

Carvalho F 380

Casciani S 503

Cason AL 937

Castellani C 93 (SR), 543 (SR)

Catane R 288

Cattan D 497

Cavallini G 543 (SR)

Censier K 342 (SR)

Cézard J-P 179

Chaigne D 770

Chakraborty R 39 (SR)

Chalas C 493

Chamaillard M 179

Chantot-Bastaraud S 452

Chardenoux S 185

Chattopadhyay P 760

Chaves FJ 959

Chelly J 493

Chen C-H 931

Chen L-H 931

Chi C-S 931

Chompret A 288

Christensen S 179

Chung J 527

Ciccodicola 155

Ciclitira PJ 828

Circolo D 155

Cisneros GJ 265

Claussen U 879 (SR) 
Clemens PR 516

Clementi M 503

Clerget-Darpoux F 590, 828

Cloostermans T 30 (SR)

Cocci F 671

Codina-Pascual M 879 (SR)

Cohn DH 265

Collins AR 437

Colombel J-F 179

Colombo MG 671

Comi GP 896 (SR)

Compain-Nouaille S 816 (SR)

Conte I 155

Conti E 349

Cooper RS 271 (SR)

Cordaux R 253

Cornelisse CJ 633

Correa P 380

Cortesi L 210

Costa MdC 872

Coutinho P 808 (SR)

Couvert P 493

Cox DW 315

Crimi M 896 (SR)

Croucher PJP 6 (SR)

Cuelenaere K 57 (SR)

Cui F 714 (SR)

Cundy T 840

Curb D 271 (SR)

Curtis AM 237

Cybulski C 955

Cymerman U 279

D'Adamo AP 585

D'Adamo P 121

D'Eustacchio A 121

Dahl N 189

Dallapiccola B 349, 433, 972 (SR)

Dall'Amico R 503

Danieli GA 69 (SR)

Dargie HJ 659

Darlow BA 840

Darlu P 794

David L 380

Day INM 437

Day LB 437

Dêbniak T 955

de Bock GH 633

De Braekeleer M 585

de Graaff E 409

de Jong DJ 884 (SR)

de Jong PTVM 215

de Knijff P 845

de Kovel CGF 884 (SR)

de la Hoya M 489 (SR)

de Luna RO 835
De Marco P 245

de Paula F 923

de Pavanello RC 923

de Visser M 552 (BR)

De Vito O 597

Deber CM 279

Dechecchi MC 93 (SR)

Decruyenaere M 30 (SR), 725 (R)

DeFaire U 845

Degioanni A 794

Dehmel HJ 858

Deka R 39 (SR)

Del-Favero J 779

Delaunay J 497

Delmaghani S 816 (SR)

Demenais F 590

Demori E 297

Demyttenaere K 30 (SR)

den Heijer M 23 (SR)

den Hollander A 155

Dermaut B 547 (SR)

Desnos M 659

Dessay S 352

Devilee P 633

Dgany O 497

Diaco M 50 (SR), 550 (ER)

Dieplinger H 693

Díez O 489 (SR)

Digilio MC 349

Di lorio E 121

di Masi A 297

Dizier M-H 590

Dobyns WB 527

Doheny K 835

Dollfus H 770

Dom R 30 (SR)

Donaudy F 121

Donnai D 468

Dorent R 659

Dowling J 527

Drira M 185

Droesch S 679

Dubreuil C 475

Duchier J 475

Ducroq D 966 (SR)

Dufier J-L 966 (SR)

Dunleavey L 437

Dupont C 452

Durán M 489 (SR)

Durban M 325

Dworniczak B 913 (L)

Egozcue J 325, 754, 879 (SR)

Ehrbrecht A 643

Einarsdottir E 81 (SR)
Eisenberg S 288

Ejarque I 959

Ekici $A B 170$

Engels H 643

Epplen JT 573

Erdem S 102 (SR), 551 (ER)

Erler A 304

Evers-Kiebooms G 30 (SR)

Faivre L 700 (SR)

Faiyaz-Ul-Haque M 77 (SR), 623 (SR)

Fan R 125

Färkkilä M 112

Farrall M 395

Federico M 210

Feilotter H 951

Félix TM 718 (SR)

Fellous M 497

Férec C 585

Ferguson-Smith MA 315

Ferrari S 210

Ferreirinha F 872

Ficarella R 585

Finkel Y 179

Finnell RH 245

Finnilä S 652

Fiorani O 802

Foncin J-F 597

Forrest SM 779

Fortunato G 342 (SR)

Franke B 884 (SR)

Fransen E 744

Frants RR 845

Frébourg T 700 (SR)

Frenzel H 6 (SR)

Frigo G 69 (SR)

Fryns J-P 30 (SR), 85 (SR), 145

Fuchs S 858

Funahashi H 364

Galbiati S 896 (SR)

Galjaard R-JH 409

Gangemi S 433

Gao S 275 (SR), 723

García-García AB 959

Garcia-Miñaur S 892

Gardner M 237

Garrett C 872

Gasbarrini G 50 (SR), 550 (ER)

Gaspar I 872

Gasparini P 121, 585

Gasser T 138

Gassull M 179

Gécz J 639 (PG)

Gehlken U 629 (SR)

Gerber S 966 (SR)
Ghorbel AM 185

Gillberg C 189

Gillessen-Kaebasch G 551 (ER)

Gillessen-Kaesbach G 201, 858

Girard T 342 (SR)

Gläser D 913 (L)

Godard B 561

Gomez-Lira M 93 (SR), 543 (SR)

Goodship J 851

Gorlova OY 425

Górski B 955

Gough SCL 840

Gow PJ 840

Gower-Rousseau C 179

Grabowski M 138

Granizo JJ 489 (SR)

Greco L 828

Greco M 503

Green RA 840

Greiwe M 858

Gribaa M 770

Grifone N 349

Grimard D 585

Grinberg I 527

Gronwald | 955

Gross A 527

Gschwend JE 17 (SR)

Guilloud-Bataille M 590

Guimarães ] 808 (SR)

Guimarães L 808 (SR), 872

Gut I 416 (SR)

Hadj-Rabia S 700 (SR)

Haeussler J 17 (SR)

Hagoel L 725 (R)

Hakiki S 966 (SR)

Haley CS 819

Hall RJ 840

Halle D 288

Hallqvist A 89 (SR)

Halme L 112

Halonen K 866

Hamann U 464

Hampe J 6 (SR)

Hanein S 966 (SR)

Haque S 623 (SR), 812 (SR)

Harada D 916

Haraldsson S 81 (SR)

Hardt C 573

Harmon DL 237

Harrison AA 840

Hauben El 611

Hautmann RE 17 (SR)

Heams T 493

Heard RNS 509

Heeringa J 225 
Heijmans BT 845

Heil SG 23 (SR)

Heinonen S 232

Heister AJGAM 884 (SR)

Helderman-van den Enden ATJM 975 (BR)

Heliö T 112

Heller A 879 (SR)

Hemminki E 565

Hemminki K 207 (L)

Hendry MA 892

Herbeth B 679

Herkommer K 17 (SR)

Hernández-Sánchez J 819

Hesse V 858

Hetmanski JB 835

Heutink P 409

Heward JM 840

Heyer E 597

Heytens L 342 (SR)

Highton J 840

Hiltunen M 232

Hinkel GK 201, 551 (ER)

Hinrichs F 629 (SR)

Hirtzlin I 475

Hodaňová K 145, 369

Hoeltzenbein M 201, 551 (ER)

Hofman A 225

Hogendoorn PCW 611

Hol FA 884 (SR)

Holden KR 937

Holder-Espinasse M 457

Holgate ST 619

Holinski-Feder E 913 (L)

Hollox EJ 911 (L)

Holmberg D 81 (SR)

Holmberg M 81 (SR)

Holmgren G 81 (SR)

Hoogendoorn WE 633

Hoovers JMN 643

Hopkins PM 342 (SR)

Hopwood P 725 (R)

Horn D 858

Horowitz M 369

Horsthemke B 555, 913 (L)

Hou N 714 (SR)

Houlston RS 665

Houten SM 196

Houwing-Duistermaat JJ 225

Hřebíček M 369

Hu B 516

Hu X 215

Huang M (SR)

Hugot J-P 179

Hukki J 265

Hul WV 457

Hunt PJ 840
Hunt S 271 (SR)

Huot T 288

Huse K 6 (SR)

Ikeguchi Y 937

Imai T 364

Inglehearn CF 420 (SR)

Isacson R 288

Ivanov VP 444

lyengar S 760

Jabs EW 718 (SR)

Jafri H 420 (SR)

Jakubowska A 955

Jallinoja P 565

Jamal SM 623 (SR)

Janićijević B 535

Jansen B 475

Janssen B 913 (L)

Januário C 872

Jazbec J 97 (SR)

Jeřábková M 145

Jenkins T 705 (SR)

Jennings CE 840

Jern C 603

Johannesson T 189

Johansson M 189

Johnson RM 279

Joly Y 561

Jood K 603

Jouannet P 493

Julian-Reynier C 725 (R)

Jurkat-Rott K 342 (SR)

Kääriäinen H 265

Kalscheuer V 138, 643

Kalscheuer VM 201

Kalscheurer VM 551 (ER)

Kamnasaran D 315

Kan D 271 (SR)

Kanasaki H 402

Kaplan J 966 (SR)

Karino K 402

Kavamura MI 64 (SR)

Kawai K 364

Kayser M 304

Keavney B 395

Keegan D 237

Keen TJ 420 (SR)

Kehl HG 201, 551 (ER)

Kelly Y 237

Kemmeren PPCW 57 (SR)

Kere J 265

Kerjean A 493

Khen M 497

Kidd JR 760

Kidd KK 760

Kilimann MW 516
Kılınç MO 851

Kim WH 6 (SR)

Kimonis VE 527

Kirk K 638 (L)

Kittler R 304

Kivisild T 535

Klarić IM 535

Kleinle S 913 (L)

Klijn JGM 633

Klinger W 342 (SR)

Kluft C 845

Kluijtmans LAJ 23 (SR)

Kmoch S 145

Knapp M 637

Knoppers BM 561

Kocaefe YÇ 102 (SR), 551 (ER) Leunissen JAM 57 (SR)

Kochhan L 913 (L)

Koenig M 770

Koillinen H 265

Komajda M 659

Kone-Paut I 50 (SR), 550 (ER)

König P 693

Kontula K 112, 888

Kosztolányi G 375

Kowalska E 955

Kozak-Ribbens G 342 (SR)

Krakow D 265

Krasemann E 913 (L)

Krasnov T 497

Kraus C 170, 913 (L)

Krawczak M 6 (SR)

Kreuz FR 858

Kristensen T 416 (SR)

Kristensen VN 416 (SR)

Krivosic R 342 (SR)

Kroisel P 913 (L)

Krol-Warmerdam EMM 633

Kronenberg F 693

Kunze J 858

Kurioka H 402

Kurtz F 17 (SR)

Laasanen J 232

Ladenvall P 603

Lagier-Tourenne C 770

Lahermo P 112

Laitinen PJ 888

Lakenberg N 845

Lambert D 679

Lammi L 866

Lampropoulos B 945

Lancet D 497

Landegren U 357

Lanz M 629 (SR)

Lanzara C 585

Lao O 385

Lappalainen M 112
La Regina M 50 (SR), 550 (ER)

Lathrop M 185, 816 (SR)

Ławniczak M 955

Leahy DT 237

Leal SM 77 (SR), 623 (SR), 812 (SR)

Lecce R 64 (SR)

Ledbetter DH 527

Lee $\mathrm{H}-\mathrm{F} 931$

Legius E 85 (SR)

Lemainque A 185, 816 (SR)

Leonard DGB 275 (SR), 723

Lesage $S 179$

Lestingi M 155

Letarte M 279

Levy-Lahad E 288

Leys A 215

Lhotta K 693

Li X 207 (L)

Lich C 913 (L)

Liehr T 879 (SR)

Lievers KJA 23 (SR)

Lima B 923

Limborska SA 444

Limburg M 737

Lind L 81 (SR)

Lisa A 802

Liston WA 892

Liu X 464

Lobato de Faria P 475

Löfgren A 547 (SR)

Löfgren-Burström A 81 (SR)

Loos HS 555

Lorenz-Depiereux B 138

Loureiro L 872

Lu T 6 (SR)

Lubinski J 955

Lubs HA 937

Luieczorek D 551 (ER)

Luijten JAFM 737

Luisetti M 553 (L)

Luke A 271 (SR)

Lv Y-X 714 (SR)

Maciel P 872

Mackay DS 784

Macry J 179

Magalhães M 872

Magalhães P 872

Magri C 802

Majamaa K 652

Majava M 185

Majeuski F 201, 858

Majewski F 551 (ER)

Majewski J 145

Mak BS-C 931 
Malvehy J 288

Manfredi S 671

Mangino M 433

Manna R 50 (SR), 550 (ER)

Mannermaa A 232

Männikkö M 185, 265

Manning P 840

Mao C-M 714 (SR)

Maraschio P 297

Margreiter R 693

Marinaki A 145

Marino B 349

Markham VH 840

Marseglia GL 297

Martin CL 527

Martin NG 845

Martinez F 937

Martins S 808 (SR)

Mascheretti S 6 (SR)

Masetti S 671

Masmoudi S 185

Mason S 503

Matamá T 808 (SR)

Mateu E 385

Matthijs G 85 (SR), 145

Maumenee IH 420 (SR)

Mavrogiannis LA 892

Maxwell D 749

Mayosi BM 395

McDonagh T 659

McGarvey ST 39 (SR)

McGaughran JM 468

McIntosh I 835

McKibbin M 420 (SR)

McLean L 840

Méhes K 375

Meinecke P 201, 551 (ER), 858

Meitinger T 138

Melchionda S 121

Melegh B 375

Melkoniemi M 265

Mělková Z 369

Mendel-Hartvig M 357

Menkiszak J 955

Mennicke K 858

Merello E 245

Merla A 687

Merlino MV 433

Merriman ME 840

Merriman TR 840

Mikulich Al 444

Millen KJ 527

Mingarelli R 349, 433, 972 (SR)

Miterski B 573

Miyazaki K 402

Moala J 185
Moati L 497

Modigliani R 179

Mohamed MD 420 (SR)

Molon A 710 (SR)

Monnier N 342 (SR)

Montagna P 710 (SR)

Montague S 179

Moraine C 352

Morar B 705 (SR)

Moroni A 245

Mosby T 835

Moses SW 516

Mueller CR 342 (SR)

Muenke M 315

Mukherjee N 760

Mulligan CJ 39 (SR)

Mundlos S 858

Munnich A 966 (SR)

Mura C 585

Murakumo Y 364

Murdolo M 64 (SR)

Murer L 503

Musante L 201, 551 (ER)

Myers R 271 (SR)

Naber AHJ 884 (SR)

Nádasi E 375

Nagaya M 364

Nakao A 364

Naluai AT 828

Namba A 744, 916

Narod SA 955

Nava A 69 (SR)

Navarro J 325, 879 (SR)

Nebel A 705 (SR)

Neri G 64 (SR)

Neumann LM 858

Nicaud V 659

Nieminend CP 866

Nietzel A 879 (SR)

Nigro V 923

Nikolaus S 6 (SR)

Nilsson-Ardnor S 81 (SR)

Nilsson M 357

Nilsson S 603, 828

Ninis VN 851

Nishikawa M 364

Nivoche Y 342 (SR)

Njajou OT 225

Noiato M 69 (SR)

Nordenskjöld M 89 (SR)

Notarnicola C 50 (SR), 550 (ER)

Nucera G 50 (SR), 550 (ER)

Nunn KP 945

Oates A 468

O'Brien PC 315
Occhi G 69 (SR)

O'Donoghue D 237

Odunsi A 760

Ogilvie CM 749

Ogino S 275 (SR), 723

Ohtsuka A 744

Okonofua F 760

O'Leary C 237

Olender T 497

Oliver-Bonet M 879 (SR)

Olivier D 163

Ollikainen V 112

Olshen RA 271 (SR)

Olthoff D 342 (SR)

Ondrová L 145

Oostra BA 409

O'Morain C 237

Opitz J 352

Opitz JM 64 (SR)

Orsmark C 189

Osborne RH 225

Osorio A 489 (SR)

Ott J 145

Ozaki T 402

Ozguc M 551 (ER)

Özgüç M 102 (SR)

Paavola-Sakki P 112

Paessler M 275 (SR), 723

Paiss T 17 (SR)

Pakstis AJ 760

Palanduz S 858

Paldi A 493

Pals G 737

Parfrey NA 237

Pariente A 497

Parik J 535

Partanen J 828

Passos-Bueno MR 718 (SR)

Patthy L 744

Patuzzo C 93 (SR)

Pearce SH 840

Pearson PL 737

Pecile V 297

Pedersen NL 845

Peek R 215

Pegg AE 937

Pegoraro E 710 (SR)

Peixoto A 380

Pellestor F 337

Pembrey M 352

Penha-Goncalves C 81 (SR)

Pepe G 503

Perandones C 835

Peretz H 288

Perez-Lezaun A 475

Peričíć M 535
Perrault I 966 (SR)

Perri C 597

Perri F 687

Peters G 288

Petit C 185, 816

Pfister M 679

Pham TL 812 (SR)

Piepoli A 687

Pignatti PF 93 (SR), 543 (SR), 553 (L)

Pihlajamaa T 265

Pipiras E 452

Pirinen S 866

Pizzuti A 349

Plendl H 913 (L)

Plomp A 215

Pocheshkhova EA 444

Podnar T 97 (SR)

Pogoda TV 444

Poirier K 493

Poirier O 659

Poirot C 493

Pokorny V 840

Pols HAP 225

Pomponi MG 64 (SR)

Pongratz D 516

Ponthieux A 679

Porteous MEM 892

Préaubert N 475

Procopio A 50 (SR), 550 (ER)

Pronk JC 737

Propping P 643

Pugh EW 835

Pugliese M 155

Puig JG 145

Puig S 288

Pujo A-L 163

Pujol A 325, 879 (SR)

Punnonen K 232

Purmann S 913 (L)

Quane KA 237

Quesneville H 590

Quintana-Murci L 802

Raap AK 643

Raffoux C 794

Rafique MA 623 (SR)

Rajmil O 754

Ramocki MB 527

Ramos L 808 (SR)

Rampazzo A 69 (SR)

Rannan-Eliya SV 892

Rashid Y 420 (SR)

Raskin S 39 (SR)

Raso A 245

Råstam M 189

Rauch A 170 
Rautenstrauss B 170

Rautio J 265

Raynaud M 352

Read AP 468

Real JT 959

Realpe L 380

Regateiro F 872

Reis A 170

Reis C 380

Rigoli L 433

Rinkel GJE 737

Rivard SR 585

Rizza A 671

Rizzoni G 503

Robinson RL 342 (SR)

Rodeck B 858

Rodríguez-López R 489 (SR)

Roetto A 585

Roizès G 659

Romano C 352

Romppanen E-L 232

Ronce N 352

Rootsi S 535

Ropers H-H 201, 551 (ER)

Rosengren A 603

Rozet J-M 966 (SR)

Rudan I 535

Rudan P 535

Rueffert H 342 (SR)

Ruiz A 288

Russell JD 945

Safro M 288

Sagramoso C 93 (SR)

Saha N 253

Salpietro DC 433

Sampson AP 619

Sander G 913 (L)

Santachiara-Benerecetti S 802

Sarafan-Vasseur N 700 (SR)

Sarkozy A 349

Savarirayan R 639 (PG)

Sayers I 619

Schollen E 85 (SR)

Schoumans J 89 (SR)

Schreiber S 6 (SR)

Schroers A 516

Schwanitz G 643

Schwartz CE 937

Schwartz K 659

Schwartz M 547 (SR)

Schweiger S 201, 551 (ER)

Schwinger E 629 (SR)

Sciacco M 896 (SR)

Scott RJ 955

Scott RS 840

Šebesta I 145
Segovia M 835

Selinger-Leneman H 590

Semino O 802

Sequeiros J 808 (SR), 872

Seven M 851

Seynaeve CMJC 633

Shafir R 288

Shalev H 497

Shalmon L 497

Shanahan F 237

Shete S 425

Shiels A 784

Shin YS 516

Shu S-G 931

Silingardi V 210

Silva F 380

Silveira I 808 (SR)

Silvestri C 210

Silvestri G 770

Simard H 585

Simard R 585

Simensen RJ 937

Simmonds A 145

Simon J 475

Simpson I 437

Sinn H-P 464

Sirajuddin SM 253

Skinner C 937

Skladny H 913 (L)

Skovlund E 416 (SR)

Slagboom PE 845

Smits APT 409

Smyth DJ 840

Snieder H 845

Sobolev V 288

Söderström I 81 (SR)

Sohail M 77 (SR), 623 (SR)

Sollid LM 828

Soodyall H 705 (SR)

Sorensen MV 444

Sorrentino V 342 (SR)

Soucek P 416 (SR)

Sousa A 872

Spadoni E 297

Speed W 760

Speevak MD 951

Spitsyn VA 444

Spitzer E 913 (L)

Splendore A 718 (SR)

Splitt M 749

Spranger M 629 (SR)

Spranger S 629 (SR)

Ståhl Y 89 (SR)

Stanziale P 687

Starke H 879 (SR)

Starling A 923

Starzyńska T 955
Steland A 573

Stevenson RE 937

Stewart GJ 509

Stibůrková B 145

Stofko JA 265

Stoll M 6 (SR)

Stoneking M 253, 304

Stopar-Obreza M 97 (SR)

Storti S 671

Strom TM 138

Struycken PM 737

Suchiman HED 845

Sun G 39 (SR)

Suzuki H 916

Swan H 888

Takahashi K 402, 744

Takahashi M 364

Tamary H 497

Tan E 102 (SR), 551 (ER)

Tandoi C 349

Tanejo K 337

Tanguy G 966 (SR)

Tanke HJ 643

Tanner SL 945

Tanzarella C 297

Tapper W 437

Tarskaya LA 444

Tartaglia S 503

Tassabehji M 468

Taylor BJ 840

Tegazzin V 342 (SR)

Teixeira A 380

Templado C 754

ten Brink J 215

Tenconi R 503

Tentler D 189

Teutsch SM 509

Thamm-Mücke B 913 (L)

Thesleff I 866

Thiel CT 170

Thiene G 69 (SR)

Thomas G 179

Thompson E 639 (PG)

Thulliez M 497

Tiepolo L 297

Tinschert S 201, 551 (ER), 858

Tiret L 659

Tlili A 185

Toiviainen H 565

Tolarova MM 835

Tollenaar RAEM 633

Tolun A 851

Torres R 145

Touitou I 50 (SR), 550 (ER)

Toupance B 597

Towbin J 69 (SR)
Tranebjćrg L 770

Trebušak-Podkrajšek K 97 (SR)

Trijbels FJM 23 (SR)

Trneny M 416 (SR)

Tsai C-R 931

Tsukamoto K 916

Tuerlings JHAM 409

Turchetti D 210

Türkmen S 858

Turner ST 271 (SR), 425

Turunen U 112

Tüysüz B 851

Tysk C 179

Uğur SA 851

Ulmer HU 464

Unis L 543 (SR)

Urwin RE 945

Usami S-i 916

Usami-ichi S 744

Utermann G 693

Vaessen N 225

Vainzof M 923

Vale J 872, 808 (SR)

Valldecabres C 959

van Asperen CJ 611, 633

Van Broeckhoven C 547 (SR)

van Broekhoven C 342 (SR)

Vandenbroucke JP 611

van den Berg JSP 737

van den Born LI 215

VanderKolk CA 835

van Driel MA 57 (SR)

van Duijn CM 225

Van Goethem G 547 (SR)

Van Marck E 611

van Ommen GJB 845

Varon R 297

van Soest S 215

van Woerden CS 196

Varon-Mateeva R 913 (L)

Vaughan P 237

Vega A 489 (SR)

Veiga A 325

Verbenko DA 444

Verdyck P 457

Vergeer J 225

Verhoef P 23 (SR)

Verloes A 452

Vermeulen M 737

Vesel S 97 (SR)

Victor J-M 179

Vieira N 923

Villems R 535

Vincent M-C 163

Vinceti M 210 
Visscher PM 819

Visser B 475

Vissing J 547 (SR)

Visvikis S 679

Vogel W 17 (SR)

Vogler GP 845

Voigt R 629 (SR)

von der Malsburg C 555

Vorgerd M 516

Vrolijk H 643

Vylet'al P 145

Wahlström J 189, 828

Wajid M 812 (SR)

Wallin A 89 (SR)

Wanders RJA 196

Wang NW 425

Wang T-Y 931

Wang $Y 714$ (SR)

Wang Y-L 714 (SR)

Warburton CP 749

Warman ML 265

Warter JM 770
Waterham HR 196

Watkins H 395

Weil D 185

Weil D (SR)

Weinhäusel A 913 (L)

Weirich H 913 (L)

Welkenhuysen M 725 (R)

Westerveld A 737

White S 643

Whitfield JB 845

Wieczorek D 201, 555 (ER)

Wiegant J 643

Wijburg FA 196

Wijmenga C 737

Wijnholds J 215

Wilcken B 945

Wilkie AOM 892

Willemen JJHT 884 (SR)

Williams B 337

Williams GD 475

Williamson R 779

Willis JA 840

Wilson RB 275 (SR), 723
Wolf J-P 452

Wood TC 937

Wörner S 17 (SR)

Wu X 271 (SR)

Würtz RP 555

Wuyts W 457

Wyszynski DF 835

Xiong M 125

Yakobson E 288

Yamamoto LU 923

Yan K 812 (SR)

Yang S-H 931

Yang S-K 6 (SR)

Yang X 714 (SR)

Yaniv I 497

Yao J-Y 714 (SR)

Ye S 437, 619

Yeoman S 840

Yonehara T 402

Young SS 951

Yuge I 744
Zackai EH 315

Zahn S 643

Zaizov R 497

Zanocco-Marani T 210

Zatz M 923

Zei G 802

Zeiger JS 835

Zeinali S 816 (SR)

Zelante L 687

Zhong Y 39 (SR)

Zhu H 245

Zhu X 271 (SR)

Zikánová M 145

Zimprich A 138

Zina ZB 185

Ziviello C 155

Zollino M 64 (SR)

Zouali H 179

Zschieschang P 858

Zuccarello D 433

Zühlke CH 629 (SR) 